\title{
Estratégia Híbrida Combinando Angioplastia Carotídea e Cirurgia Cardiovascular
}

\author{
Carlos Miguel Fava1', Leon R. Valdivieso1, Gustavo A. Lev¹, Lorena G. Villagra1', Gaspar Caponi', \\ Antonio Semiglia' ${ }^{1}$, Eduardo Torres ${ }^{1}$, Oscar A. Mendiz ${ }^{1}$
}

\section{RESUMO}

Fundamentos: Lesões carotídeas estão presentes em $8 \%$ a $14 \%$ dos candidatos a cirurgia cardiovascular e aumentam o risco de acidente vascular cerebral perioperatório. Esse problema é particularmente importante em pacientes com doença coronária e/ou valvar grave que não são candidatos a procedimentos de revascularização em momentos diferentes. Avaliamos os resultados de uma estratégia híbrida de tratamento, na qual angioplastia carotídea e cirurgia cardiovascular foram realizadas de forma sequencial, com intervalo de algumas horas. Método: Foram tratadas lesões carotídeas $\geq 70 \%$ em pacientes sintomáticos e $\geq 80 \%$ nos assintomáticos. Ácido acetilsalicílico foi administrado antes da angioplastia carotídea e heparina, no momento do procedimento. Finalizada a intervenção percutânea, os pacientes foram transferidos para a sala de cirurgia para realização do procedimento cardiovascular. Clopidogrel foi administrado habitualmente 8 horas após o término da cirurgia. Resultados: Foram incluídos 43 pacientes consecutivos tratados com a estratégia híbrida. Predominaram pacientes do sexo masculino $(81 \%)$, com média de idade de 70,5 $\pm 8,5$ anos, sendo $25,6 \%$ diabéticos. Foi utilizado sistema de proteção cerebral em 42 pacientes e implantado stent em todos. Cirurgia de revascularização miocárdica (CRM) foi realizada em 20 pacientes, CRM + substituição de valva aórtica (SVA) em 18, CRM + cirurgia da aorta ascendente em 1 , CRM + plástica da valva mitral em 1, CRM + substituição de valva mitral (SVM) em 1, SVA em 1, e SVM em 1. Os resultados hospitalares demonstraram 4 óbitos (2 por falência de múltiplos órgãos, 1 por insuficiência cardíaca e 1 por sangramento), 1 caso de infarto agudo do miocárdio perioperatório e nenhum caso de acidente vascular cerebral. Acompanhamento tardio foi realizado em todos os pacientes aos $25 \pm 15$ meses. Após a alta hospitalar, ocorreram duas mortes, não relacionadas aos procedimentos percutâneo ou cirúrgico, e nenhum evento neurológico. Conclusão: A estratégia híbrida combinando angioplastia carotídea e cirurgia cardiovascular é factível

\author{
ABSTRACT \\ Hybrid Strategy Combining Carotid Stenting \\ and Cardiovascular Surgery
}

Background: Carotid lesions are observed in $8 \%$ to $14 \%$ of cardiovascular surgery candidates and increase the risk of perioperative stroke. This is particularly important in patients with coronary disease and/or severe valve disease who are not candidate to revascularization procedures at different time points. We assessed the results of a hybrid strategy, where carotid angioplasty and cardiovascular surgery were performed sequentially with an interval of a few hours. Method: Carotid lesions $\geq 70 \%$ in symptomatic patients and $\geq 80 \%$ in asymptomatic patients were treated. Acetylsalicylic acid was administered prior to carotid stenting and heparin was administered at the time of the procedure. Once the percutaneous intervention was over, patients were transferred to the surgery room to carry out the cardiovascular procedure. Clopidogrel was administered 8 hours after the end of the surgery. Results: Forty-three consecutive patients were included and treated with the hybrid strategy. Male patients $(81 \%)$ were prevalent, with mean age of $70.5 \pm 8.5$ years, and $25.6 \%$ were diabetic. A cerebral protection system was used in 42 patients and stents were implanted in all of them. Coronary artery bypass graft (CABG) was performed in 20 patients, $C A B G+$ aortic valve replacement (AVR) in 18, CABG + ascending aorta repair in 1, CABG + mitral valve annuloplasty in $1, C A B G+$ mitral valve replacement (MVR) in 1, AVR in 1 and MVR in 1. Hospital results showed 4 deaths (2 due to multiple organ failure, 1 due to heart failure and 1 to bleeding), 1 case of perioperative acute myocardial infarction and no strokes. Late followup was performed in all of the patients at $25 \pm 15$ months. After hospital discharge, there were two deaths not related to the percutaneous or surgical procedures and no neurological events. Conclusion: A hybrid strategy combining carotid stenting and cardiovascular surgery is feasible and safe with an acceptable complication rate. It may be a

\footnotetext{
${ }^{1}$ Hospital Universitário Instituto de Cardiologia e Cirurgia Cardiovascular Fundação Favaloro - Buenos Aires, Argentina.

Correspondência: Carlos Miguel Fava. Avenida Belgrano, 1749 -

Capital Federal - Buenos Aires, Argentina - 1093

E-mail: cfava@ffavaloro.org

Recebido em: 18/4/2009 - Aceito em: 8/6/2009
} 
Fava CM, et al. Estratégia Híbrida Combinando Angioplastia Carotídea e Cirurgia Cardiovascular. Rev Bras Cardiol Invas. 2009; $17(2): 190-5$.

e segura, com taxa de complicações aceitável. Pode ser válida em pacientes clinicamente instáveis, sendo necessária, entretanto, a realização de estudos com maior número de doentes para reproduzir nossos achados.

Descritores: Procedimentos cirúrgicos cardiovasculares. Artéria carótida primitiva/cirurgia. Artéria carótida primitiva/ patologia. good option in clinically unstable patients, however, further studies with a large number of patients are required to reproduce our findings.

Descriptors: Cardiovascular surgical procedures. Carotid artery, common/surgery. Carotid artery, common/pathology.
A presença de lesões carotídeas graves em pacientes candidatos a cirurgia cardiovascular varia de $8 \%$ a $14 \%$ e pode ser ainda maior, de acordo com outros relatos ${ }^{1,2}$. Esses pacientes apresentam maior risco de acidente vascular perioperatório, que representa complicação grave, devastadora, incapacitante e com forte impacto social e econômico.

A angioplastia carotídea demonstrou sua utilidade nos pacientes de alto risco, quando comparada à endarterectomia carotídea ${ }^{3}$. Contudo, não há evidência clara acerca de qual é a melhor estratégia para aqueles pacientes que se encontram instáveis por doença coronária e/ou valvar, que apresentam lesão carotídea grave e que, pela condição cardiológica, não são candidatos a procedimentos de revascularização em momentos diferentes. A maioria dos estudos com essa população foi realizada em pacientes submetidos a endarterectomia carotídea e cirurgia de revascularização miocárdica em momentos diferentes, e em outros estudos os procedimentos foram realizados de forma simultânea.

O objetivo desta análise foi avaliar os resultados hospitalares e tardios dos pacientes de alto risco cirúrgico nos quais foi realizada uma estratégia híbrida combinando angioplastia carotídea e cirurgia cardiovascular.

\section{MÉTODO}

\section{Definições}

Este é um estudo retrospectivo, não-randomizado, realizado em um único centro. Entre junho de 1998 e março de 2009, foram efetuados 525 procedimentos consecutivos de angioplastia carotídea, e em 43 (8,2\%) desses pacientes foi realizada angioplastia carotídea seguida de cirurgia cardiovascular.

Os dados foram obtidos por meio da história clínica durante a internação e o seguimento foi prospectivo, com informações obtidas mediante avaliação ambulatorial, contato telefônico direto com o paciente ou por meio do médico de família.

Todos os pacientes foram hospitalizados por apresentarem angina refratária e/ou insuficiência cardíaca decorrente de doença coronária e/ou doença valvar grave, sem condições clínicas de realizar as intervenções cardíaca e carotídea em momentos distintos. Os pacientes com doença coronária apresentavam lesões em dois ou três vasos, não passíveis de serem resolvidas por angioplastia.

A doença carotídea foi avaliada por meio de ultrassonografia com Doppler e confirmada pela angiografia de vasos do pescoço. Foram tratadas lesões $\geq 70 \%$ em pacientes sintomáticos e $\geq 80 \%$ nos assintomáticos. A angioplastia carotídea foi realizada com sistema de proteção distal de forma rotineira a partir de 1999, quando ficou disponível na Argentina. Todos os pacientes receberam implante de stent.

Os pacientes foram tratados previamente à angioplastia carotídea com ácido acetilsalicílico $(200$ mg/dia via oral) e $10.000 \mathrm{UI}$ de heparina sódica no momento do procedimento. Em procedimentos com duração superior a uma hora, os pacientes receberam $3.000 \mathrm{UI}$ adicionais de heparina sódica. Finalizada a angioplastia carotídea, os pacientes foram transferidos do laboratório de hemodinâmica para a sala de cirurgia para realização do procedimento cirúrgico cardiovascular programado.

O clopidogrel, iniciado logo depois de descartado sangramento maior, foi aplicado por via oral ou por sonda nasogástrica, caso o paciente estivesse ainda em assistência respiratória mecânica (habitualmente depois de 8 horas da chegada à unidade de tratamento intensivo).

O sucesso técnico da angioplastia carotídea foi definido como a obtenção de obstrução residual < 30\% após o implante do stent. Todos os pacientes foram submetidos a exame neurológico pré e pós-procedimento, e em caso de suspeita de complicação neurológica foi realizada tomografia computadorizada do cérebro.

O acidente vascular cerebral foi definido como "menor" quando ocorresse déficit com aparecimento posterior à angioplastia carotídea $\leq 4$ pontos na escala de acidente vascular cerebral do National Institute of Health (NIH) ou com recuperação total dentro de 7 dias. Acidente vascular cerebral "maior" foi considerado na ocorrência de déficit $>4$ pontos (incapacitante). Ataque isquêmico transitório foi considerado como déficit focal com recuperação total $<24$ horas.

Todos os déficits neurológicos ipsilaterais que ocorreram após a angioplastia carotídea, anteriores ou pos- 
Fava CM, et al. Estratégia Híbrida Combinando Angioplastia Carotídea e Cirurgia Cardiovascular. Rev Bras Cardiol Invas. 2009; $17(2): 190-5$.

teriores à cirurgia cardiovascular, foram atribuídos à angioplastia carotídea, enquanto os déficits neurológicos contralaterais, observados após a cirurgia cardiovascular, foram atribuídos à cirurgia.

Óbito, infarto do miocárdio e acidente vascular cerebral foram analisados na fase hospitalar e no seguimento tardio. Infarto do miocárdio foi definido como ocorrência de novas ondas Q no eletrocardiograma, como aumento do valor sérico da creatina quinase fração $M B(C K-M B)$ (> $50 \mathrm{Ul} / \mathrm{ml}$ nos casos de cirurgia de revascularização miocárdica sem circulação extracorpórea, > $80 \mathrm{UI} / \mathrm{ml}$ naquelas com circulação extracorpórea ou $>100 \mathrm{UI} / \mathrm{ml}$ nos procedimentos valvulares ou combinados), como aparecimento de bloqueio de ramo esquerdo novo e permanente ou, ainda, como aparecimento de novas áreas com déficit segmentar da contratilidade no ecocardiograma. Foi considerada assistência respiratória prolongada aquela $>24$ horas pós-cirurgia e insuficiência cardíaca pós-cirúrgica quando se detectasse índice cardíaco $<2,2 \mathrm{l} / \mathrm{m}$, em uso de inotrópicos (milrinona ou mais de $6 \mathrm{~g} / \mathrm{kg} / \mathrm{min}$ de dobutamina), por mais de duas horas.

A angioplastia carotídea foi realizada por acesso femoral com anestesia local; a cirurgia cardiovascular foi realizada com anestesia geral, utilizando-se oxigenador de membrana para circulação extracorpórea, e proteção miocárdica (anterógrada e/ou retrógrada sanguínea ou cristaloide). A escolha da técnica para realização de angioplastia carotídea e cirurgia cardiovascular ficou a cargo do operador.

Todos os pacientes assinaram o consentimento informado para a angioplastia carotídea e para a cirurgia cardiovascular correspondente.

Foram utilizados números absolutos e porcentagens para descrever a população e os resultados, bem como o valor médio e o desvio padrão para as variáveis contínuas.

\section{RESULTADOS}

\section{Características da população}

Foram incluídos 43 pacientes consecutivos, submetidos a angioplastia carotídea e cirurgia cardiovascular em estratégia combinada. Predominaram pacientes do sexo masculino (81\%), com média de idade de 70,5 \pm 8,5 anos, $13,9 \%$ com idade igual ou superior a 80 anos, $25,6 \%$ eram diabéticos e 6,9\% haviam sido previamente submetidos a cirurgia cardiovascular. Em relação aos antecedentes neurológicos, 9,3\% tinham acidente vascular cerebral prévio, $11,6 \%$ apresentavam oclusão carotídea contralateral e um paciente apresentava reestenose pós-endarterectomia carotídea.

Lesões carotídeas graves bilaterais foram observadas em 6 pacientes, dos quais 4 estavam clinicamente instáveis, decidindo-se realizar, nesses pacientes, an- gioplastia carotídea bilateral (no início da série) e cirurgia cardiovascular combinadas. Os 2 pacientes restantes se encontravam estáveis, optando-se em um por angioplastia carotídea e no outro por endarterectomia carotídea; após a primeira intervenção, esses pacientes apresentaram condição cardíaca instável em período inferior a 30 dias, decidindo-se pela realização de angioplastia carotídea contralateral e cirurgia cardiovascular combinadas, passando então a integrar nossa casuística.

Pouco mais da metade dos pacientes $(55,8 \%)$ apresentava quadro clínico de síndrome coronária aguda refratária ao tratamento farmacológico e os restantes, insuficiência cardíaca (48,8\%), angina crônica estável e insuficiência cardíaca $(2,3 \%)$, e aneurisma de aorta ascendente (2,3\%).

A função ventricular esquerda, prévia à cirurgia cardiovascular, tinha comprometimento grave $(\leq 30 \%)$ em 13 pacientes e moderado (30-40\%) em outros 3. Em relação à gravidade angiográfica, 20 pacientes apresentavam lesão de tronco de artéria coronária esquerda, 27 apresentavam lesão em três vasos, 5 apresentavam lesão de dois vasos e 9, lesão em um vaso.

O escore de Parsonnet calculado foi de $12 \pm 4$ e o EuroSCORE, de 7,6 $\pm 2,2$. A mortalidade esperada pelo EuroSCORE foi de 10,4 $\pm 7,4 \%$ (Tabela 1 ).

\section{Angioplastia carotídea e cirurgia cardiovascular}

Realizou-se angioplastia carotídea em 47 artérias carótidas. Foi implantado stent em todas as lesões, sendo 24 de nitinol (Precise ${ }^{T M}$ em 12 pacientes, Protege ${ }^{T M}$ em 5, Smart ${ }^{\mathrm{TM}}$ em 4 e X-ACT ${ }^{\mathrm{TM}}$ em 3) e 23 Carotid Wallstent ${ }^{\mathrm{TM}}$. Foi utilizado sistema de proteção cerebral em 42 pacientes (42 filtros, 3 sistemas de balão oclusor distal e um de balão oclusor proximal). Esses sistemas de proteção cerebral não foram utilizados no primeiro caso da série, pois não se encontravam disponíveis no País.

A cirurgia cardiovascular realizada consistiu em cirurgia de revascularização miocárdica em 20 pacientes, cirurgia de revascularização miocárdica mais substituição de valva aórtica em 18, cirurgia de revascularização miocárdica mais cirurgia de aorta ascendente em 1, cirurgia de revascularização miocárdica mais plástica de valva mitral em 1, cirurgia de revascularização miocárdica mais substituição de valva mitral em 1, substituição de valva aórtica em 1 e substituição de valva mitral em 1 . Duas cirurgias foram realizadas sem circulação extracorpórea (Tabela 2).

\section{Resultados hospitalares}

Obteve-se sucesso técnico da angioplastia carotídea em todos os pacientes e nenhum paciente apresentou eventos clínicos (óbito, acidente vascular cerebral maior ou menor, ataque isquêmico transitório ou infarto agudo do miocárdio) depois do procedimento (Tabela 3). Um paciente apresentou evidência ultrassonográfica de trombo intrastent imediatamente após a cirurgia, em avalia- 
Fava CM, et al. Estratégia Híbrida Combinando Angioplastia Carotídea e Cirurgia Cardiovascular. Rev Bras Cardiol Invas. 2009; $17(2): 190-5$.

TABELA 1 Características clínicas

\begin{tabular}{lc}
\hline & $\mathbf{n}(\%)$ \\
\hline Número de pacientes & 43 \\
Idade (anos) & $70,5 \pm 8,5$ \\
$\geq 80$ anos & $6(13,9)$ \\
Sexo masculino & $35(81,4)$ \\
Diabetes melito & $11(25,6)$ \\
Hipertensão arterial sistêmica & $35(81,4)$ \\
Claudicação intermitente & $11(25,6)$ \\
Acidente vascular cerebral prévio & $4(9,3)$ \\
Ataque isquêmico transitório prévio & $3(7)$ \\
Infarto agudo do miocárdio prévio & $12(27,9)$ \\
Cirurgia cardiovascular prévia & $3(7)$ \\
$\quad$ Segunda cirurgia cardíaca & $1(2,3)$ \\
Oclusão carotídea contralateral & $5(11,6)$ \\
Clínica & \\
Síndrome coronária aguda & $34(79)$ \\
Insuficiência cardíaca & $21(48,8)$ \\
Angina crônica/insuficiência cardíaca & $1(2,3)$ \\
Aneurisma da aorta sintomático & $1(2,3)$ \\
Vasos coronários doentes & \\
Tronco da artéria coronária esquerda & $20(46,5)$ \\
3 vasos & $27(62,8)$ \\
2 vasos & $5(11,6)$ \\
1 vaso & $9(20,9)$ \\
Cunção ventricular & \\
Comprometimento grave & $13(30,2)$ \\
Engioplastia carotídea bilateral & $4(9)$ \\
EuroSCORE & $7,6 \pm 2,2,2$ \\
\hline &
\end{tabular}

TABELA 2 Cirurgia cardiovascular realizada

\begin{tabular}{lc}
\hline & $\mathbf{n}(\%)$ \\
\hline CRM & $20(46,5)$ \\
CRM + substituição de valva aórtica & $18(41,9)$ \\
CRM + cirurgia da aorta ascendente & $1(2,3)$ \\
CRM + plástica da valva mitral & $1(2,3)$ \\
CRM + substituição de valva mitral & $1(2,3)$ \\
Substituição de valva aórtica & $1(2,3)$ \\
Substituição de valva mitral & $1(2,3)$ \\
\hline
\end{tabular}

CRM = cirurgia de revascularização miocárdica.

ção de controle, sem apresentar sinais neurológicos; esse trombo desapareceu nas ultrassonografias carotídeas posteriores. Esse paciente foi classificado como um ataque isquêmico transitório.
TABELA 3

Resultados hospitalares

\begin{tabular}{lc}
\hline & $\mathbf{n}(\mathbf{\%})$ \\
\hline Sucesso técnico & $43(100)$ \\
Sucesso clínico & $39(90,7)$ \\
Acidente vascular cerebral (maior ou menor) & --- \\
Infarto agudo do miocárdio & $1(2,3)$ \\
Óbito relacionado à angioplastia carotídea & --- \\
Óbito total & $4(9,3)$ \\
\hline
\end{tabular}

Após a cirurgia cardiovascular, 4 pacientes faleceram no hospital (3 submetidos a cirurgia de revascularização miocárdica mais substituição de valva aórtica e 1 a cirurgia de revascularização miocárdica), 2 deles em decorrência de falência de múltiplos órgãos, 1 por insuficiência cardíaca e 1 por sangramento (esse paciente apresentava duas cirurgias cardíacas prévias, tendo sido realizadas outras duas cirurgias para estancar sangramento pós-cirúrgico). O escore de Parsonnet desses pacientes era de 16, 17, 18 e 19.

As seguintes complicações pós-cirúrgicas foram detectadas: insuficiência cardíaca com a exigência de inotrópicos em $26(60,4 \%)$ pacientes, comprometimento da função renal em $13(30,2 \%)$, assistência respiratória prolongada em $10(23,2 \%)$, necessidade de assistência circulatória com balão de contrapulsação em 7 (16,3\%), sangramento de causa cirúrgica em $6(13,9 \%)$, reoperação por diálise em $5(11,6 \%)$, sepse em $5(11,6)$, e infarto agudo do miocárdio perioperatório em $1(2,3 \%)$.

\section{Acompanhamento tardio}

O acompanhamento tardio foi realizado em todos os pacientes aos $25 \pm 15$ meses (7-72 meses), dos quais 33 tinham mais de 12 meses de seguimento. Entre a alta hospitalar e o final do primeiro ano, não ocorreram mortes, eventos neurológicos ou reestenose carotídea. No seguimento superior a um ano, ocorreram duas mortes de causas não relacionadas aos procedimentos percutâneo ou cirúrgico e nenhum evento clínico neurológico ou reestenose carotídea.

\section{DISCUSSÃo}

Em decorrência da maior expectativa de vida da população, o grupo de pacientes que chegam instáveis ao hospital e não respondem adequadamente ao tratamento médico, exigindo cirurgia de emergência, crescerá substancialmente nos próximos anos, e representará desafio real à equipe de saúde, já que apresenta maior morbidade e mortalidade e, consequentemente, maiores custos do tratamento.

A angioplastia carotídea desenvolveu-se extraordinariamente nos últimos anos graças à tecnologia dos novos stents, aos sistemas de proteção cerebral e 
Fava CM, et al. Estratégia Híbrida Combinando Angioplastia Carotídea e Cirurgia Cardiovascular. Rev Bras Cardiol Invas. 2009; $17(2): 190-5$.

à curva de aprendizado dos operadores, com consequente melhora dos resultados.

Hertzer et al. ${ }^{2}$ encontraram que $28 \%$ dos doentes em quem foi realizada endarterectomia carotídea apresentavam lesões coronárias graves, sendo a doença coronária a causa mais frequente de morte após endarterectomia carotídea.

O risco perioperatório de acidente vascular cerebral da cirurgia cardiovascular é $<2 \%$ quando há estenose carotídea menor que 50\%, de $10 \%$ quando a estenose oscila entre $50 \%$ e $80 \%$, de $11 \%$ a $19 \%$ quando supera $80 \%$, e de $20 \%$ nos que apresentam oclusões totais ou lesões bilaterais não tratadas ${ }^{4}$. Risco de acidente vascular cerebral e de morte em pacientes com lesão carotídea crítica em quem foi realizada apenas cirurgia de revascularização miocárdica foi relatado em $11,5 \%{ }^{5}$.

Naylor et al. ${ }^{6}$ analisaram os pacientes em quem foi realizada endarterectomia carotídea e cirurgia cardiovascular simultânea ou postergada. No primeiro grupo, os pacientes apresentaram maior mortalidade e infarto perioperatório, mas o segundo grupo apresentou maior taxa de acidente vascular cerebral. O risco de morte/acidente vascular cerebral/infarto foi de 11,5\% vs. $10,2 \%$, sem apresentar diferença significativa entre essas estratégias.

Evagelopoulus et al. ${ }^{7}$ estudaram 313 pacientes em quem foi realizada endarterectomia carotídea e cirurgia de revascularização miocárdica simultâneas. A média de idades foi de 66 anos. Do total de pacientes, 26,2\% apresentavam lesão de tronco de artéria coronária esquerda; 43,5\%, diminuição da fração de ejeção do ventrículo esquerdo; $48 \%$, lesão carotídea bilateral; e $7,7 \%$, oclusão contralateral. A taxa de infarto agudo do miocárdio perioperatório foi de 3,2\%, de acidente vascular cerebral foi de $2,2 \%$, e de morte, $8,9 \%$.

Em outro estudo, Kolh et al. ${ }^{8}$ relataram uma série de 311 pacientes submetidos a endarterectomia carotídea e cirurgia cardiovascular simultâneas, realizadss entre 1989 e 2002. Óbito ocorreu em 6\%, acidente vascular cerebral em $4 \%$, ataque isquêmico transitório em $2 \%$, déficit neurológico reversível em 1,5\%, e infarto perioperatório em 7\%. A análise multivariada detectou calcificação proximal da aorta, doença vascular periférica e procedimentos de emergência como preditores independentes de eventos adversos.

Fareed et al. ${ }^{9}$ realizaram uma meta-análise na qual incluíram 324 pacientes de 12 estudos em quem foi realizada endarterectomia carotídea e cirurgia de revascularização miocárdica sem circulação extracorpórea. O risco de morte foi de 1,5\%; de morte e acidente vascular cerebral ipsilateral, de 1,6\%; de morte e acidente vascular cerebral total, de 2,2\%; e de morte, infarto agudo do miocárdio e acidente vascular cerebral, de $3,6 \%$. A menor taxa de eventos adversos foi provavel- mente decorrente da menor manipulação da aorta na cirurgia sem circulação extracorpórea.

Em nosso estudo, o principal problema a ser enfrentado era a realização de cirurgia cardiovascular sob dupla antiagregação plaquetária, caso fosse realizada em etapas, e isso resultaria em risco aumentado de sangramento. Por outro lado, a realização em etapas nem sempre é possível em decorrência da grave condição cardiovascular. Por esse motivo, os pacientes só receberam ácido acetilsalicílico e heparina nãofracionada antes do procedimento carotídeo, sendo imediatamente transferidos para a sala de cirurgia. A dupla agregação foi iniciada depois da cirurgia, quando o sangramento maior foi controlado.

Van der Heyden et al. ${ }^{10}$ analisaram 356 pacientes com lesão carotídea assintomática, nos quais foi realizada angioplastia carotídea e cirurgia cardiovascular em dois momentos. O tempo médio entre a angioplastia carotídea e a cirurgia foi de 22 dias (1 a 3 meses). Utilizou-se sistema de proteção distal em 40\% dos pacientes. A taxa de complicações depois da angioplastia carotídea foi: morte de causa cardíaca, 0,3\%; acidente vascular cerebral, 1,4\% (acidente vascular cerebral maior, $0,3 \%$; acidente vascular cerebral menor, $1,1 \%$ ); ataque isquêmico transitório, 2,2\%; e infarto agudo do miocárdio não-fatal, 0,6\%. As complicações associadas à cirurgia cardiovascular foram: morte, 3,4\% (cardíaca, 2\%; neurológica, 0,3\%; não-neurológica e não-cardíaca, $1,1 \%$ ); acidente vascular cerebral, 1,7\% (acidente vascular cerebral maior, $0,8 \%$; acidente vascular cerebral menor, 0,6\%); ataque isquêmico transitório, 1,4\%; e infarto não-fatal, 1,4\%. A taxa de morte/acidente vascular cerebral foi de $4,8 \%$ e de morte/acidente vascular cerebral/infarto agudo do miocárdio não-fatal foi de 6,7\%.

Há outros relatos nos quais se realizou angioplastia carotídea e cirurgia cardiovascular em dois momentos com resultados encorajadores, mas esses resultados não podem ser transpostos para nossa população, já que o perfil de gravidade é diferente, sendo muito maior em nossos pacientes ${ }^{11,12}$.

Chiarello et al. ${ }^{13}$ realizaram angioplastia carotídea e cirurgia de revascularização miocárdica simultânea em 10 pacientes, nos quais não foram observados morte ou acidente vascular cerebral.

Previamente, em 30 pacientes submetidos a angioplastia carotídea e cirurgia cardiovascular simultâneas pelo nosso grupo, não foi observado acidente vascular cerebral nem após a angioplastia carotídea nem após a cirurgia, e óbito foi registrado em 3 pacientes, não relacionado à angioplastia ${ }^{14}$.

Esta série atual analisada apresenta várias limitações, entre as quais se destacam: série de um só hospital, com operadores selecionados e experientes; e estudo não-randomizado, retrospectivo e sem grupo controle. No entanto, nosso estudo destaca-se por ser 
Fava CM, et al. Estratégia Híbrida Combinando Angioplastia Carotídea e Cirurgia Cardiovascular. Rev Bras Cardiol Invas. 2009; $17(2): 190-5$.

um dos poucos publicados com pacientes que necessitavam não só de cirurgia de revascularização miocárdica, mas também de cirurgia de revascularização miocárdica associada à substituição valvar $(48,8 \%)$ e de cirurgia valvar isolada.

Em decorrência da falta de evidências claras nesta população, acreditamos que sejam necessários estudos randomizados e controlados com maior volume de doentes. Nesse grupo de pacientes, a decisão da melhor estratégia deve ser particularizada, após avaliação de um grupo multidisciplinar integrado por cardiologistas, neurologistas, intervencionistas e cirurgiões cardiovasculares.

Adicionalmente, consideramos que, nos pacientes estáveis, deve ser realizada revascularização carotídea e cirurgia cardiovascular em dois momentos diferentes, e naqueles que não podem esperar a estratégia híbrida apresenta-se como uma opção.

\section{CONCLUSÃO}

A realização de uma estratégia híbrida combinando angioplastia carotídea e cirurgia cardiovascular foi factível e segura, sem apresentar complicações após o procedimento percutâneo, especialmente acidente vascular cerebral ou infarto, e com boa evolução no seguimento tardio. Essa estratégia híbrida pode ser válida nos pacientes clinicamente instáveis, sendo necessário, entretanto, realizar estudos com maior número de doentes para reproduzir nossos achados.

\section{CONFLITO DE INTERESSES}

Os autores declararam inexistência de conflito de interesses.

\section{REFERÊNCIAS BIBLIOGRÁFICAS}

1. Ricotta JJ, Faggioli GL, Castilone A, Hassett JM. Risk factors for stroke after cardiac surgery: Buffolo Cardiac-Cerebral Study Group. J Vasc Surg. 1995;21(2):359-64.

2. Hertzer NR, Young JR, Beven EG, Graor RA, O'Hara PJ, Ruschhaupt WF $3^{\text {rd }}$, et al. Coronary angiography in 506 patients with extracranial cerebrovascular disease. Arch Intern Med. 1985;145(5):849-52.
3. Yadav JS, Wholey MH, Kuntz RE, Fayad P, Katzen BT, Mishkel GJ, et al. Protected carotid-artery stenting versus endarterectomy in high risk patients. N Engl J Med. 2004; 351(15):1493-501.

4. Eagle KA, Guyton RA, Davidoff R, Ewy GA, Fonger J, Gardner TJ, et al. ACC/AHA guidelines for coronary artery bypass graft surgery: executive summary and recommendations: A report of the American College of Cardiology/American Heart Association Task Force on Practice Guidelines (Committee to revise the 1991 guidelines for coronary artery bypass graft surgery). Circulation. 1999;100(13):1464-80.

5. Das SK, Brow TD, Pepper J. Continuing controversy in the management of concomitant coronary and carotid disease: an overview. Int J Cardiol. 2000;74(1):47-65.

6. Naylor AR, Cuffe RL, Rothwell PM, Bell PR. A systematic review of outcomes following staged and synchronous carotid endarterectomy and coronary artery bypass. Eur J Vasc Endovasc Surg. 2003;25(5):380-9.

7. Evagelopoulos N, Trenz M, Beckmann A, Krian A. Simultaneous carotid endarterectomy and coronary artery bypass grafting in 313 patients. Cardiovasc Surg. 2000;8(1) 31-40.

8. Kolh PH, Comte L, Tchana-Sato V, Honore C, Kerzmann A, Mauer $\mathrm{M}$, et al. Concurrent coronary and carotid artery surgery: factors influencing perioperative outcome and longterm results. Eur Heart J. 2006;27(1):49-56.

9. Fareed KR, Rothwell PM, Mehta Z, Naylor AR. Synchronous carotid endarterectomy and off-pump coronary bypass: an updated, systematic review of early outcomes. Eur J Vasc Endovasc Surg. 2009;37(4):375-8.

10. Van der Heyden J, Suttorp MJ, Bal ET, Ernst JM, Ackerstaff RG, Schaap J, et al. Staged carotid angioplasty and stenting followed by cardiac surgery in patients with severe asymptomatic carotid artery stenosis: early and long-term results. Circulation. 2007;116(18):2036-42.

11. Kovacic JC, Roy PR, Baron DW, Muller DW. Staged carotid artery stenting and coronary artery bypass graft surgery: initial results from a single center. Catheter Cardiovasc Interv. 2006; 67(1):142-8.

12. Randall MS, McKevitt FM, Cleveland TJ, Gaines PA, Venables GS. Is there any benefit from staged carotid and coronary revascularization using carotid stents? A single-center experience highlights the need for a randomized controlled trial. Stroke. 2006;37(2):435-9.

13. Chiarello L, Tomai F, Zeitani F, Versaci F. Simultaneous hybrid revascularization by carotid stenting and coronary artery bypass grafting. Ann Thorac Surg. 2006;81(5):1883-5.

14. Mendiz O, Fava C, Valdivieso L, Dulbecco E, Raffaelli $H$, Lev G, et al. Synchronous carotid stenting and cardiac surgery: an initial single-center experience. Catheter Cardiovasc Interv. 2006;68(3):424-8 